\title{
Is Intraoperative Leak Test Required in Laparoscopic Sleeve Gastrectomy?
}

\author{
Burhan Mayir \\ Department of General Surgery, Antalya Training and Research Hospital, Antalya, Turkey
}

\begin{abstract}
Objective: To investigate the efficacy of intraoperative leak test (IOLT) by methylene blue to identify the presence of leak during standardised sleeve gastrectomy (SG) procedure.

Study Design: Cross-sectional comparative study.

Place and Duration of Study: Department of General Surgery, Antalya Training and Research Hospital, Antalya, Turkery, between January 2017 and December 2018.

Methodology: A total of 226 consecutive sleeve gastrectomised patients, on whom IOLT was not performed, were included in the study, and were named as group 1. In order to compare with these patients, 226 consecutive sleeve gastrectomised patients, on whom IOLT was performed, were named as group 2. The groups were compared for postoperative leak, other complications, and mortality.

Results: In group 1, leakage was detected in two patients. Although IOLT was negative in all patients of group 2, leakage was detected in two patients. The leakage rate (1.6\%) was similar in both groups. In IOLT group, sensitivity and positive predictive value of IOLT was $0 \%$; and negative predictive value was $99.1 \%$.

Conclusion: The routine use of an IOLT did not reduce the incidence of postoperative leak. IOLT should not be routinely performed, rather it would be appropriate for selected patients.
\end{abstract}

Key Words: Laparoscopic sleeve gastrectomy, Intraoperative leak test, Stapler line leak.

How to cite this article: Mayir B. Is Intraoperative Leak Test Required in Laparoscopic Sleeve Gastrectomy? J Coll Physicians Surg Pak 2021; 31(03):318-321.

\section{INTRODUCTION}

With the increase in incidence of obesity and obesity-related metabolic problems in the world, the rate of bariatric-metabolic surgery is also increasing. There are different types of procedures, but the most common surgical procedure is sleeve gastrectomy (SG). ${ }^{1}$ Although sleeve gastrectomy is a restrictive surgery, it has been shown to be effective not only in the treatment of obesity but also in the treatment of obesity-related comorbid diseases. ${ }^{2}$

Sleeve gastrectomy has been performed worldwide for a long time. However, the SG technique is not fully standardised, and there are still many controversial technical issues. One of these issues is the usage of intraoperative leak test (IOLT). ${ }^{3}$

Leak after SG is one of most feared complication and may be fatal. ${ }^{4}$ Some authors advocated that leak can be detected earlier by IOLT, so can be treated immediately.

Correspondence to: Dr. Burhan Mayir, Department of General Surgery, Antalya Training and Research Hospital, Antalya, Turkey

E-mail: burmay@yahoo.com

Received: April 20, 2020; Revised: October 14, 2020;

Accepted: November 18, 2020

DOI: https://doi.org/10.29271/jcpsp.2021.03.318
But in literature, there is no standardised approach for the necessity of IOLT, for the type of test to be performed and for timing of the test. Although studies about usage of IOLT at colorectal surgery and RYGB have shown the efficacy of test, the efficacy at SG has not been clearly demonstrated. However, many surgeons still continue to perform IOLT in SG. ${ }^{5}$

The aim of this study is to investigate the efficacy of IOLT by methylene blue to identify the presence of leak for SG procedure.

\section{METHODOLOGY}

Patients that have undergone sleeve gastrectomy were included in the study with body mass index more than 40 and also having at least one obesity-related comorbide disease or the ones with body mass index more than $35 \mathrm{Kg} / \mathrm{m}^{2}$. Patients who have undergone re-sleeve gastrectomy were excluded. Ethics Committee approval was obtained for the study.

A total of 226 consecutive sleeve gastrectomised patients on whom IOLT was not applied, were included in the study, named as group 1. In order to compare with these patients, 226 consecutive sleeve gastrectomised patients on whom IOLT was applied, were named as group 2. In author's experience, formerly one applied IOLT by methylene blue during SG routinely, this practice was abandoned after March 2018. 
Table I: Patients demographics. IQR: (25th percentile - 75th percentile).

\begin{tabular}{|l|c|c|}
\hline & Group 1 & Group 2 \\
\hline Age (median IQR) & $41.0(31.0-39.0)$ & $39.0(30.0-47.0)$ \\
\hline Sex (women / men) & $176(77.9 \%) / 50(22.1 \%)$ & $182(80.5 \%) / 44(19.5 \%)$ \\
\hline Length(cm) (median IQR) & $163.0(158.0-168.0)$ & $163.0(158.0-168.0)$ \\
\hline Kilo (kg) (Median IQR) & $125.0(115.0-130.0)$ & $122.0(110.8-135.0)$ \\
\hline BMI (median IQR) & $46.5(42.8-48.5)$ & 0.487 \\
\hline
\end{tabular}

Table II: Peroperatuar datas of patients. IQR: (25th percentile - 75th percentile).

\begin{tabular}{|l|c|c|}
\hline & Group 1 & Group 2 \\
\hline Duration of operation (median IQR) & $51.5(44.0-61.0)$ & $65.0(58.0-80.0)$ \\
\hline Lenght of (median IQR) & $3.0(3.0-3.0)$ & $<0.001$ \\
\hline
\end{tabular}

Table III: IOLT efficiency to predict postoperative leak.

\begin{tabular}{|l|c|c|c|}
\hline & Positive postoperative leak & Negative postoperative leak & Total \\
\hline Positive IOLT & 0 & 0 & 0 \\
\hline Negative IOLT & 2 & 224 & 226 \\
\hline Total & 2 & 224 & 226 \\
\hline
\end{tabular}

During study design, this alteration was used for definition of groups. In the end of December 2018, patients' data were collected retrospectively and demographic information, surgical findings, postoperative follow-up and complications were recorded.

All patients underwent sleeve gastrectomy with the same surgical technique by the one general surgeon, experienced at bariatric-metabolic surgery. Dissection started with opening of the greater omentum using $5 \mathrm{~mm}$ vessel sealing device. The dissection then continued towards the gastroesophageal junction. The left crus was then completely freed of any attachments to avoid leaving a posterior pouch, when constructing the sleeve in this region. After insertion of 38 French gastric calibration tube, gastric transection was started $2 \mathrm{~cm}$ proximal to the pylorus using $60 \mathrm{~mm}$ endo-stapler. The following staplers were placed approximately $1 \mathrm{~cm}$ from the calibration tube in the direction of the gastroesophageal junction. After completing the transection, bleeding points were secured using $10 \mathrm{~mm}$ endoclips. The transected stomach was then removed through the $12 \mathrm{~mm}$ left midclavicular port. After removal of transected stomach, just for patients in group 2, pylorus was laparoscopically closed with a bowel clamp, stomach was filled with approximately $60 \mathrm{cc}$ of methylene blue, given through the orogastric tube. The presence of leak from the staple line was investigated. Drain was placed next to the staple line routinely for both groups. Postoperative imaging or CT scans for detection of leak were not applied routinely. All patients were started clear liquid diet on postoperative day one. The drains of all patients were removed on postoperative day 7 . All patients followed by surgeon at postoperative day 7 ; and $1,3,6,12^{\text {th }}$ month.

The primary outcome of study was presence of leak. The presence of methylene blue at staple line during the operation, radiologically revealed contrast extravasation from staple line post-operatively, and observation of saliva in the drain were defined as leak. Secondary outcomes were post- operative complications, including bleeding and infectious complications and mortality rate.

All statistical analyses were performed with SPSS version 22 (IBM Corporation, Chicago, IL). Qualitative data were expressed as numbers and percentages, while quantitative as median (Interquartile range, IQR). Comparisons of categorical variables between groups were conducted using the Pearson's Chi-square test. Differences between groups in non-normally distributed continuous variables were tested using the Mann-Whitney U-test. The $p$ value $<0.05$ was accepted as statistically significant. Sensitivity and specificity analysis of the IOLT were also performed, and positive and negative predictive value calcuated.

\section{RESULTS}

Group 1 consists of 176 (77.9\%) females, 50 (22.1\%) males; and group 2 consists of 182 (80.5) females and 44 (19.5\%) males. All patients were operated laparoscopically. Demographic characteristics of the patients were similar (Table I). In group 1, leakage was detected in two (1.6\%) patients. Both were in the fundus. Leakage was detected at the postoperative day 8 and 40 in these patients. Although IOLT was negative in all patients of group 2, leakage was detected in two $(1.6 \%)$ patients at postoperative day 5 . One of them was in the fundus and the other was in the antrum. The leakage rate was similar in both groups.

Postoperative bleeding, which led hemodynamic instability was observed at six patients, three from each group. Re-operation was not required for these patients, as conservative management with blood transfusions was succesful. No other complications or mortality were observed at both groups.

For both groups, IOLT results had been given in Table II. In IOLT group, sensitivity and positive predictive value of IOLT were $0 \%$ and negative predictive value was $99.1 \%$ (Table III). 


\section{DISCUSSION}

Leakage after SG is an important complication which can be seen in $0-8 \%$ of the patients, which can lead to prolonged hospital stay, sepsis, chronic fistula, multiorgan failure or even death. ${ }^{6,7}$ To reduce the incidence of postoperative leaks, many surgeons perform IOLT with methylene blue. Alternative test can be done by air insufflation. ${ }^{8}$

Most of the leakages occur a few days after surgery, as it was observed in this study patients. ${ }^{5}$ Therefore, IOLT is ineffective for detection of leakage that will appear a few days after surgery. IOLT may be useful for detection of leakages due to defects in surgical procedures. In the SG, the entire stapler line constructed with linear staplers and both the anterior or posterior stapler lines can be evaluated visually and if present, technical problem on stapler line can be seen. On the other hand, gastrojejunostomy in Roux-en-Y gastric bypass, colonic anastamosis or esophagojejunostomy are constructed by circular stapler, so posterior side of anastamosis cannot be evaluated visually. Thus, an IOLT may be useful for evaluating the posterior side of anastamosis for leakage. ${ }^{8-11}$ But in SG, IOLT will not give any additional information if the stapler line is visually robust. For this reason, IOLT should not be performed routinely, it would be appropriate for selected patients.

In literature, there is no statement for usage of IOLT in SG. ${ }^{12}$ In 2012, the International Sleeve Gastrectomy Expert Panel Consensus Statement did not provide a definite suggestion for IOLT, but the $48 \%$ of the participants recommended IOLT. ${ }^{12}$ Recent consensus published in the Netherlands, reported that IOLT was not considered to be key step. ${ }^{13}$ However, IOLT is still widely used in SG. ${ }^{14}$

In this study, there were two cases of leakage, although there were no positive IOLT among 226 patients. Overall, we found that sensitivity and positive predictive value of IOLT are $0 \%$. These data are consistent with the findings of the other studies. Sethi et al. used IOLT in 1,329 patients and did not observe any intraoperative leakage. But at postoperative follow-up, leakage was observed in 15 patients. Sensivity of IOLT was found as $0 \%$. In this study, it was reported that despite leakage, IOLT was still negative at reoperation of two patients. ${ }^{15}$ At a multicentric study of Bingham et al., sensitivity of IOLT was $8.7 \% .{ }^{16}$ In a study, Kirby et al., detected postoperative leakage at three of 224 patients, who underwent SG; but none of these patients had leakage during IOLT. ${ }^{17}$ Parikht et al. concluded that performance of IOLT did not seem to impact leakage detection rate in a meta-analysis. ${ }^{18}$

IOLT with methylene blue may have some negative impacts. In addition to low sensivity of IOLT for detection of leakage, IOLT may cause leakage. In the IOLT, inflating the stomach with methylene blue can cause tension in the new staple line, and it can cause deterioration in healing. ${ }^{19}$ This may be the reason for the higher rates of leakage after IOLT in some studies. Also IOLT increases both operation time and cost of surgery. In addition, although methylene blue is used widely in various surgical procedures, it can cause anaphylaxis in some patients. ${ }^{20}$

In this study, unlike similar studies in the literature, all surgeries were performed with the same surgical technique by the one general surgeon experienced at bariatric-metabolic surgery. Thus, the error that may occur due to different technical variations is minimised.

This study has some limitations. Firstly, it has some unfavourable effects as being a retrospective study. Secondly, the minor leak without any clinical significance was not evaluated.

\section{CONCLUSION}

Routine IOLT sensitivity is $0 \%$, and it is not associated with decrease in incidence of leakage after SG. In this study, intraoperative leakage was not observed in any patient on whom we have performed IOLT with methylene blue; but leakage was detected later in the postoperative period. According to the results of this study, IOLT should not be routinely performed, it would be appropriate for selected patients.

\section{ETHICAL APPROVAL:}

Ethics Committee approval was obtained for the study.

\section{PATIENTS' CONSENT:}

Informed consents were obtained from all patients.

\section{CONFLICT OF INTEREST:}

The author declared no conflict of interest.

\section{AUTHOR'S CONTRIBUTION:}

BM: Design of the work, the acquisition, analysis or interpretation of data for the work, drafting the work.

\section{REFERENCES}

1. Gagner M, Hutchinson C, Rosenthal R. Fifth International consensus conference: Current status of sleeve gastrectomy. Surg Obes Relat Dis 2016; 12(4):750-6. doi: 10.1016/j.soard.2016.01.022.

2. Schauer PR, Bhatt DL, Kashyap SR. Bariatric surgery versus intensive medical therapy for diabetes. N Engl J Med 2014; 371(7):680-2. doi: 10.1056/NEJMc1407393.

3. Abdallah E, El Nakeeb A, Youssef T, Abdallah H, Ellatif MA, Lotfy $A$, et al. Impact of extent of antral resection on surgical outcomes of sleeve gastrectomy for morbid obesity (a prospective randomised study). Obes Surg 2014; 24(10):1587-94. doi: 10.1007/s11695-014-1242-x.

4. Iossa A, Abdelgawad M, Watkins BM, Silecchia G. Leaks after laparoscopic sleeve gastrectomy: overview of pathogenesis and risk factors. Langenbecks Arch Surg 2016; 401(6):757-66. doi: 10.1007/s00423-016-1464-6.

5. Kim J, Azagury D, Eisenberg D, DeMaria E, Campos GM. 
American society for metabolic and bariatric surgery clinical Issues committee. ASMBS position statement on prevention, detection, and treatment of gastrointestinal leak after gastric bypass and sleeve gastrectomy, including the roles of imaging, surgical exploration, and nonoperative management. Surg Obes Relat Dis 2015; 11(4):739-48. doi: 10.1016/j.soard.2015.05.001.

6. Gagner M, Brown M. Update on sleeve gastrectomy leak rate with the use of reinforcement. Obes Surg 2016; 26(1):146-50. doi: 10.1007/s11695-015-1899-9.

7. Arteaga-González I, Martín-Malagón A, Martín-Pérez J, Carrillo-Pallarés A. Usefulness of clinical signs and diagnostic tests for suspected leaks in bariatric surgery. Obes Surg 2015; 25(9):1680-4. doi: 10.1007/s11695- 015-1589-7.

8. Bingham J, Lallemand $M$, Barron M, Kuckelman J, Carter P, Blair $\mathrm{K}$, et al. Routine intraoperative leak testing for sleeve gastrectomy: Is the leak test full of hot air? Am J Surg 2016; 211(5):943-7. doi: 10.1016/j.amjsurg.2016.02.002.

9. Celik S, Almalı N, Aras A, Yılmaz O, Kızıltan R. Intraoperatively testing the anastamotic integrity of esophagojejunostomy using methylene blue. Scand J Surg 2017; 106(1): 62-7. doi: 10.1177/1457496916630652.

10. Shin RB. Intraoperative endoscopic test resulting in no postoperative leaks from the gastric pouch and gastrojejunal anastomosis in 366 laparoscopic Roux-en-Y gastric bypasses. Obes Surg 2004;14(8):1067-9. doi: 10.1381/ 0960892041975613.

11. Smith S, McGeehin W, Kozol RA, Giles D. The efficacy of intraoperative methylene blue enemas to assess the integrity of a colonic anastomosis. BMC Surg 2007; 7:15. doi: 10.1186/1471-2482-7-15.

12. Rosenthal RJ, International sleeve gastrectomy expert panel, Diaz AA, Arvidsson D, Baker RS, Basso N, Bellanger $D$, et al. International sleeve gastrectomy expert panel consensus statement: Best practice guidelines based on experience of $>12,000$ cases. Surg Obes Relat Dis 2012; 8(1):8-19. doi: 10.1016/j.soard.2011.10.019.
13. Kaijser MA, van Ramshorst GH, Emous M, Veeger NJGM, van Wagensveld BA, Pierie J. A Delphi consensus of the crucial steps in gastric bypass and sleeve gastrectomy procedures in the Netherlands. Obes Surg 2018; 28(9):2634-43. doi: 10.1007/s11695-018-3219-7.

14. Lin S, Guan W, Hans P, Liang H. Status of Iaparoscopic sleeve gastrectomy in China: A national survey. Obes Surg 2017; 27(11):2968-73. doi: 10.1007/s11695-017-2727-1.

15. Sethi M, Zagzag J, Patel K, Magrath M, Somoza E, Parikh MS, et al. Intraoperative leak testing has no correlation with leak after laparoscopic sleeve gastrectomy. Surg Endosc 2016; 30(3):883-91. doi: 10.1007/s00464-015-4286-7.

16. Bingham J, Kaufman J, Hata K, Dickerson J, Beekley A, Wisbach $G$, et al. A multicenter study of routine versus selective intraoperative leak testing for sleeve gastrectomy. Surg Obes Relat Dis 2017; 13(9):1469-1475. doi: 10.1016/j.soard.2017.05.022.

17. Kirby GC, Macano CAW, Nyasavajjala SM, Sahloul M, Nijjar $R$, Daskalakis $M$, et al. The birmingham experience of high-pressure methylene blue dye test during primary and revisional bariatric surgery: A retrospective cohort study. Ann Med Surg (Lond) 2017; 23:32-4. doi: 10.1016/j.amsu. 2017.09.015.

18. Parikh M, Issa R, McCrillis A, Saunders JK, Ude-Welcome A, Gagner M. Surgical strategies that may decrease leak rate after laparoscopic sleeve gastrectomy: A systematic review and meta-analysis of 9991 cases. Ann Surg 2013; 257(2):231-7. doi: 10.1097/SLA.0b013e31826cc714.

19. Causey MW, Fitzpatrick E, Carter P. Pressure tolerance of newly constructed staple lines in sleeve gastrectomy and duodenal switch. Am J Surg 2013; 205(5):571-4 discussion 574-575. doi: 10.1016/j.amjsurg.2012.12.008.

20. Ozdemir A, Mayir B, Demirbakan K, Oygur N. Efficacy of methylene blue in sentinel lymph node biopsy for early breast cancer. J Breast Health 2014; 10(2):88-91. doi: 10.5152/tjbh.2014.1914. 